\title{
CONTROVERSY
}

Ramon M. Esclamado, MD, Section Editor

\section{MANAGEMENT OF UNKNOWN PRIMARY TUMOR}

\author{
Consultants: Doug Chepeha, MD, ${ }^{1}$ Wayne Koch, MD, ${ }^{2}$ Karen Pitman, MD ${ }^{3}$ \\ ${ }^{1}$ Department of Otolaryngology-Head and Neck Surgery, University of Michigan Medical Center, Ann Arbor, \\ Michigan \\ ${ }^{2}$ Department of Otolaryngology-Head and Neck Surgery, Johns Hopkins University, Baltimore, Maryland \\ ${ }^{3}$ Department of Otolaryngology, University of Mississippi Medical Center, Jackson, Mississippi
}

\section{CAse presentation}

A 37-year-old man is initially seen with a 4-month history of a gradually enlarging, painless rightsided neck mass. He denies any otalgia, dysphagia, odynophagia, hoarseness, fever, night sweats, or weight loss. His medical history is significant only for mild COPD, and he has smoked two packs per day for 20 years. He is married and is a right-handed police officer.

Examination of the head and neck reveals the absence of any mucosal lesions in the upper aerodigestive tract by visual inspection, bimanual palpation, and fiberoptic examination of the nasal cavity, nasopharynx, hypopharynx, and larynx. There is no serous otitis media, trismus, parapharyngeal space fullness, or cranial nerve abnormalities. Palpation of the neck shows normal parotid, submandibular, and thyroid glands. There is a $4.0 \times 3.5-\mathrm{cm}$ firm, nonpulsatile right level II mass that is freely mobile from the sternocleidomastoid muscle (SCM) and the carotid sheath structures.

Readers are invited to submit interesting or difficult cases to Ramon M. Esclamado, MD, Section Editor, Department of Otolaryngology and Communicative Disorders, The Cleveland Clinic Foundation, 9500 Euclid Avenue, Cleveland, Ohio 44195.

Head \& Neck 25: 499-504, 2003

Published online 21 March 2003 in Wiley InterScience

(www.interscience.wiley.com) DOI: 10.1002/hed.10287
Fine-needle aspiration biopsy is performed and reveals metastatic poorly differentiated squamous cell carcinoma.

\section{What additional preoperative diagnos- tic studies are indicated?}

Dr. Chepeha: If the fine-needle aspiration biopsy was read by an outside pathologist, the specimen will be submitted to our pathology department for evaluation. For any patient with clinical evidence of a neck metastasis from squamous cell carcinoma of the head and neck, a CT scan with contrast from skull base to the midliver is ordered. The purpose of the scan is to evaluate for an occult primary site, which can be especially useful in the nasopharynx, base of tongue, and tonsil; to determine whether the biopsy-proven node has evidence of gross extracapsular spread into surrounding structures and to evaluate the extent of nodal disease; to check for a second primary tumor in the lung or esophagus; and to assess the lung for metastatic disease.

Dr. Koch: Palpation of the palatine and lingual tonsil is a very useful and underused diagnostic procedure. Although this may be done most revealingly in the operating room after a muscle 
relaxant has been administered, there are situations in which it should be done during the office physical examination. Digital palpation of the tongue base will elicit a gag response in most patients, but with proper preparation, it will not cause vomiting or result in a bite injury to the doctor. The tongue base is the site most frequently found to harbor an occult primary malignancy in most series of unknown primary of the upper aerodigestive tract (UADT). Many tongue base tumors are obscured from view with mirror or fiberoptic visualization because of the lumpy, irregular contour of the healthy lingual tonsil tissue and the tangential direction of view. Most small to mid-sized base of tongue (BOT) cancers are identified first by palpation in the personal experience of this author. Most patients who are told what will transpire (I must hold your tongue gently and feel the back of your throat for a moment. It will make you gag. Just continue to breathe through your nose) will willingly and successfully undergo this examination.

If the node is in the supraclavicular region, or if the histologic findings indicate a poorly differentiated malignancy or malignancy of uncertain nature, a chest and abdominal CT scans (or PET scan) are indicated.

Special immunohistochemcial staining of the FNA sample can be very useful in the workup of poorly differentiated malignancy. Markers for melanoma, mesenchymal tumors, salivary gland, lung, thyroid, prostate, colon, and other malignancies in distant sites are available and may direct further investigation.

Dr. Pitman: A CT or MRI scan with contrast from the base of skull to the thoracic inlet, a chest $\mathrm{x}$-ray, and a metabolic profile are indicated diagnostic studies. Imaging augments the physical examination of the neck and potential primary sites. Imaging can show suspicious adenopathy in other levels and anatomically characterize suspicious nodes. Because tumors of the head and neck tend to metastasize to predictable levels, the location of suspicious nodes can direct the search for the primary tumor. Extracapsular extension or suspicious adenopathy in the contralateral neck also impacts tumor staging and treatment. Imaging evaluates possible primary sites that are difficult to assess on physical examination, including clinically silent sites (eg, paranasal sinuses and submucosal areas). Because tumors of the lymphoid tissue of the pharynx often have a significant submucosal component, imaging may focus the search for the primary site. A chest $x-$ ray and metabolic laboratory profile are obtained to evaluate distant metastatic sites. Although distant metastases are unlikely in this patient, this is required for accurate tumor staging.

2. A CT scan with contrast is obtained from the skull base to the clavicles, which did not reveal any areas suspicious for a primary tumor. The neck mass was a cystic, well-circumscribed mass in right level II without involvement of the SCM or internal jugular vein. No other suspicious adenopathy was demonstrated. Would an FDG-PET scan be useful in this situation?

Dr. Koch: The sensitivity and specificity of PET scanning is still being explored in the literature. Preliminary studies indicate that it can successfully identify the primary site in some cases. It may be particularly useful in cases in which the entire body should be surveyed.

Dr. Pitman: FDG-PET has been shown to be valuable in detecting a small subset of unknown primary tumors. Studies to date that have examined the role of FDG-PET in head and neck cancer suggest that a minimum tumor volume must be present before increased glucose use is detected. This minimum tumor volume has not been reliably quantified. Because many occult primary tumors are probably very small at the time of presentation, FDG-PET may not be especially helpful in determining the location of the primary site. Prospective study of the diagnostic accuracy of FDG-PET for detecting the occult primary tumor may clarify its diagnostic usefulness.

Dr. Chepeha: Yes, its usefulness in this situation would be to reveal the primary site, a secondary primary site, or metastatic disease in the lungs. Of particular interest is whether it could be of assistance in explaining the undiscovered primary site. The usefulness of PET for imaging small tumor volumes is evolving. At present, the resolution is approximately $7 \mathrm{~mm}$. It is certainly expected that many primary lesions being worked up as an unknown primary tumor would be smaller than $7 \mathrm{~mm}$. The PET-CT scanner and the technological evolution of emission scanning should help improve the fidelity of this test. At present, if positive, the PET scan can be very helpful at detecting biopsy locations. If negative, 
an occult primary site still needs to be ruled out by random biopsies.

\section{A FDG-PET scan was obtained and reveals increased activity only in the area of the right level II node. What addition workup is indicated?}

Dr. Pitman: Additional evaluation includes examination under anesthesia, panendoscopy, palpation of the Waldeyer's ring, and directed biopsies. Clues to the site of the primary tumor are gleaned from the physical examination, imaging, location of suspicious nodes, and their radiographic and histopathologic characteristics. In this case I would biopsy the tongue base, tonsil, piriform sinus, and nasopharynx. Tonsillectomy is required for adequate histopathologic evaluation of the tonsil, and for practical reasons I perform bilateral tonsillectomy. If only the right tonsil is removed, the patient will have an asymmetric and potentially confusing examination of the oropharynx postoperatively, possibly confounding the follow-up evaluation. If all biopsy specimens are negative, repeated examinations may reveal the primary tumor. If a primary tumor is not found, the patient will need a dental evaluation and possible dental extractions before beginning radiation therapy. It is beneficial to be persistent in the search for the primary tumor, because therapy targeted at a known primary tumor is less morbid than the extensive radiation used to treat an unknown primary tumor.

Dr. Chepeha: The patients need to be taken to the operating room where the diagnostic studies are reviewed, looking for the most subtle asymmetry or irregularity, particularly in the nasopharynx, tonsil, and base of tongue, with particular attention to the portion of the upper aerodigestive tract immediately adjacent to the nodal metastasis. In this case, it would be the right tonsil and adjacent base of tongue (assuming this node is in level IIa). Evaluation under anesthesia begins with a thorough palpation of the tonsil and base of tongue. This step is very helpful in discovering occult primary lesions. Examination of the entire head and neck aerodigestive tract with a Dedo laryngoscope followed by rigid esophagoscopy to $25 \mathrm{~cm}$ and nasopharyngoscopy with a $30^{\circ}$ rigid endoscope. If, at this point, with the combination of the diagnostic studies and the physical examination, a primary lesion has not been discovered, random biopsies are per- formed. Random biopsy of both fossa of Rosenmuller, midline posterior nasopharynx, midline base of tongue, bilateral base of tongue, bilateral pyriform sinus, bilateral aryepiglottic fold should be done. If there is tonsillar tissue, I will perform an ipsilateral tonsillectomy; otherwise, I will randomly biopsy this site. If there is no evidence of a lesion within the tonsil even after excision, some surgeons will perform a bilateral tonsillectomy. I have not incorporated this approach into my practice, largely because of the published effectiveness of homolateral irradiation for tonsillar cancer. If known primary tonsillar cancer responds to homolateral radiation over hundreds of patients, I think it is unlikely that an unknown tonsillar primary tumor would metastasize to the contralateral neck.

\section{Dr. Koch: Same answer as Question 1.}

4. Subsequent examination under anesthesia and directed biopsies are negative, and the patient is staged $\mathrm{T} \times \mathrm{N} 2 \mathrm{aM} 0$. What is your treatment recommendation?

Dr. Chepeha: My treatment recommendation is based on the size and location of the node. If the node is greater than $3 \mathrm{~cm}$ and not in level IIb or V, which is the case in this clinical scenario, we recommend external beam radiation therapy followed by a neck dissection 8 weeks after completion of radiation. If the node was less than $3 \mathrm{~cm}$, we would perform a neck dissection only if there was radiologic or clinical evidence of disease 12 weeks after the completion of treatment. If the node(s) are limited to levels IIb or V, we would stage and treat the lesion as a nasopharyngeal primary tumor. Our recommendation would be concomitant cisplatinum, 5-FU, and external beam radiation. We would perform a neck dissection if there were clinical or radiologic (CT-PET) evidence of disease at 12 weeks. Neck dissection is recommended after, rather than before, radiation for the following reasons: (1) the target tissues are theoretically better oxygenated, making radiotherapy more effective; (2) a complication secondary to the surgery will not delay radiation; and (3) if a radioresistant primary tumor becomes evident after definitive radiation, it can be removed with the neck dissection in a single definitive surgery.

Dr. Koch: It is strongly recommended that "directed biopsies" include tonsillectomy in any case 
in which the palatine tonsils remain. Given an approximate $10 \%$ rate of contralateral or bilateral spread from the tonsils to the neck, both tonsils should be removed.

Treatment recommendation is for irradiation of the neck in cases in which nodal disease is (1) multifocal, (2) shows extranodal extension (on surgical pathologic evaluation of the neck dissection specimen), (3) is larger than $2 \mathrm{~cm}$ for a single metastatic focus, and (4) when the neck has been violated by incisional or excisional biopsy. Radiation of Waldeyer's ring as a likely site of an occult primary tumor is more controversial. Patients should be informed of the facts and uncertainties pertinent to this decision. Options include irradiation of all of Waldeyer's ring, irradiation of the ipsilateral portion of Waldeyer's rings shielding the contralateral parotid, or close surveillance without radiation to the pharynx. The first option results in severe xerostomia and related problems. It provides a modest improvement in locoregional disease-free survival $(<15 \%)$. With close surveillance, the emergence of a mucosal primary tumor may be detected early with an acceptable likelihood of success with standard treatment. A multi-institutional trial is underway in Europe (EORTC) comparing the first and last option. The compromise option seems appealing, providing therapy to the most likely primary-bearing tissue with limited risk of complication. Yet, it spends the radiation option while failing to cover for contralateral spread of disease. Patient age, comorbidity, reliability, smoking, and drinking history should be taken into account.

Dr. Pitman: I recommend radiation therapy to potential primary sites and both sides of the neck. Sites to be tested are the nasopharynx, oropharynx, and hypopharynx. Treatment of the contralateral neck is warranted, because all these tumor sites have a significant incidence of contralateral metastases. Although some centers may advocate treatment of the involved neck with neck dissection or ipsilateral cervical irradiation alone, this option does not address the primary tumor or contralateral neck. Studies that have critically evaluated this option have shown that unilateral neck treatment alone results in a higher rate of local and regional recurrence. I advocate radiation therapy before neck dissection. An important consideration is the timing of treatment. If surgery is performed first, treatment of the primary tumor is delayed 4 to 6 weeks. I would perform neck dissection regardless of the response of neck dis- ease to radiation. Although much controversy exists concerning the management of advanced cervical metastases, I believe the best chance for cure in this 37-year-old man includes postradiation neck dissection.

5. The patient is treated with definitive radiotherapy consisting of 200 cGy daily fractions to a total dose of $73 \mathrm{~Gy}$ to all potential primary sites, including the nasophrynx and both sides of the neck. Should the oral cavity be included in the treatment field?

Dr. Pitman: The oral cavity is not typically included in the radiation ports for an unknown primary tumor. In contrast to the lymphatic tissues of the pharynx, oral cavity sites can be adequately assessed on physical examination, and tumors are symptomatic at an early stage. This makes detection of oral cavity tumors relatively straightforward compared with sites typically associated with an unknown primary tumor (eg, base of tongue and tonsil). For these reasons it is unlikely that the primary tumor is located in the oral cavity, and excluding the oral cavity from the radiation ports will not place the patient at unnecessary risk of local recurrence. The benefit will be the decreased morbidity compared with including the oral cavity in the radiation ports.

Dr. Chepeha: No, it is unnecessary to include the oral cavity.

Dr. Koch: No.

6. Two months after the completion of radiation therapy, there is no palpable disease in either side of the neck. Is a CT or FDGPET scan useful at this point to help determine the need for a neck dissection?

Dr. Koch: Modestly. The original size of the nodal disease should be considered with planned dissection if the largest node is $>3 \mathrm{~cm}$ in diameter.

Dr. Pitman: The results of CT or FDG-PET would not impact my decision to perform postradiation neck dissection. The decision to operate is based on the advanced stage of neck disease and the potential for microscopic foci of tumor to persist after nonoperative treatment of advanced cervical metastases. These tumor foci are below the limits of resolution for CT or PET and may be present despite a clinical complete response. 
The posttreatment scan is helpful to assess the scar and fibrosis that result from treatment and provides meaningful baseline information.

Dr. Chepeha: Yes, this node is greater than 3 $\mathrm{cm}$ and I will assume in level IIa, so we would perform a neck dissection independent of radiologic findings. For nodes smaller than $3 \mathrm{~cm}$ or limited to levels IIb and V, a combined CT-PET with contrast performed 12 weeks after the completion of treatment is useful in picking up residual disease. If a CT-PET scanner is not available, the single test of most value would be a PET scan. Obtaining a PET scan 2 months after treatment increases the false-positive rate; therefore, to improve accuracy, the PET would be deferred to for an additional 4 weeks.

\section{If you elect to dissect the neck, what type of neck dissection would you perform?}

Dr. Chepeha: The current standard of care for an $\mathrm{N} 2 \mathrm{a}$ node is to perform a radical neck dissection. The spinal accessory nerve, internal jugular vein, and sternocleidomastoid should be sacrificed based on the extent of involvement as assessed by the pretreatment CT scan. In addition, if these structures seemed scarred or tethered to the lymphadenectomy specimen during surgery, they should be removed. In all cases, the accessory nerve should be grafted if removed. As part of an IRB-approved protocol, I would perform a neck dissection levels I to IV and remove the accessory nerve, internal jugular vein, and the sternocleidomastoid as per the previously described guidelines. There is a growing body of literature that suggests that this more limited lymphadenectomy is oncologically sound and has the benefit of improving shoulder function and shoulderrelated quality of life.

Dr. Koch: This should be determined by clinical stage and level of the nodal disease. For a single metastatic node less than $3 \mathrm{~cm}$ without clinical suspicion of ECS, a selective neck dissection including the involved level and contiguous level(s) may suffice. Frozen section analysis of excised nodes should be performed with extension to a comprehensive dissection if multiple histologic involved nodes are identified.

Structures removed in a comprehensive dissection depend on their degree of suspicion for ECS and location. Small, fully mobile node(s) can be removed with a functional (type III) modified radical neck dissection.

Dr. Pitman: The extent of neck dissection is another controversial topic in the management of advanced cervical metastases. Clinical data support the use of selective neck dissection after radiation therapy in select cases. This patient had no evidence of suspicious adenopathy in level I or $\mathrm{V}$ at the initial evaluation, so there is a good probability that occult metastases in these levels were treated with radiation. The involved node has also had a marked clinical response to radiation. For these reasons I believe a selective procedure is warranted. I would perform a selective neck dissection removing levels II to IV in this case.

\section{COMMENTARY}

The case presentation itself illustrates a fundamental diagnostic principle: any patient with a neck mass suspicious for a cervical nodal metastasis from an unknown primary malignancy requires a thorough history and head and neck examination, including flexible fiberoptic endoscopy, to search for a primary lesion. If none is found, FNA biopsy of the neck mass is the preferred next step, whereas open biopsy of the neck mass should be reserved for the unusual instances in which the FNA is nondiagnostic and a primary source is not found by endoscopy and/or radiologic evaluation. Dr. Pitman makes the important point that the location of the metastatic adenopathy can direct the search for the primary site, because tumors of the head and neck tend to metastasize to predictable nodal levels. Dr. Koch illustrates the importance of careful palpation of the tonsil and tongue base and the use of immunocytochemistry to assist in the workup of the poorly differentiated malignancy.

The use of ancillary radiologic studies varies among the consultants. Dr. Chepeha recommends CT imaging from the skull base to the mid-liver to gain additional important staging information. In addition, an FDG-PET scan is recommended, because a positive result is quite helpful diagnostically, with the limitation of resolution being 7 mm. Dr. Pitman uses CT/MRI from the skull base to thoracic inlet, a CXR, but infers that the diagnostic usefulness of FDG-PET is still not clarified. Dr. Koch emphasizes the importance of CT or PET scanning to evaluate infraclavicular sites when there is a high risk of distant metastasis or in unusual malignancies that may have a nonhead and neck source. 
Elimination under anesthesia (EUA) is routinely used by all the consultants, and the importance of directed biopsies is made. If tonsil tissue is present, tonsillectomy is performed, although there is disagreement regarding performance of ipsilateral or bilateral tonsillectomy. Dr. Koch states that in the situations in which EUA cannot be performed, appropriate examination can be performed in the office setting if careful palpation of the tongue base and tonsil is done as part of a complete head and neck examination.

If the primary tumor is not found, both Drs. Chepeha and Pitman recommend definitive radiation therapy to potential primary sites and both sides of the neck, with planned postradiation neck dissection in this specific patient with $\mathrm{N} 2 \mathrm{a}$ neck disease, and give excellent rationale for the treatment decision. Dr. Koch recommends neck dissection first, with radiation therapy to the most likely primary-bearing tissue and of the neck for specific indications. The consultants agree that the oral cavity can be excluded from irradiation (because of the location of the nodal disease).

In the instance in which there is a complete clinical response in the neck after definitive radiation therapy, all the consultants agree that neck dissection should still be performed when the largest node is $>3 \mathrm{~cm}$ is size, and preneck dissection imaging to evaluate the neck is not necessary. In necks in which the largest node was $<3$ $\mathrm{cm}$, Dr. Chepeha recommends CT-PET scanning, or at a minimum, PET scanning 3 months after radiation therapy to help confirm a true complete clinical response in the neck. The appropriate type of neck dissection to perform remains controversial, and the consultants make salient points regarding the controversy. For neck dissections performed before planned radiation therapy, there is a growing body of evidence supporting the efficacy of SND of appropriate levels, with judicious sacrifice of involved structures determined at the time of surgery. ${ }^{1}$ For the $\mathrm{N}+$ neck that has become a clinical and radiographic N0 neck after irradiation, there is also recent evidence suggesting the SND is also safe in this setting. ${ }^{2}$

The management of the patient with an unknown primary squamous cell carcinoma of the head and neck is being redefined as advances in technology may assist in the diagnostic evaluation and in assessing treatment response; appropriate application of selective neck dissection in the N+ neck can minimize the shoulder dysfunction and cosmetic deformity associated with modified or radical neck dissection. The consultants' comments highlight some contemporary approaches to this common problem and identify areas for active investigation so that we might minimize treatment-related morbidity while maintaining or improving survival of our patients.

\section{REFERENCES}

1. Andersen PE, Warren F, Spiro J, et al. Results of selective neck dissection in management of the node-positive neck. Arch Otolaryngol Head Neck Surg 2002;128:1180-1184.

2. Fritz MA, Esclamado RM, Lorenz RR, Wood BG, Lavertu P, Strome M. Recurrence rates after selective neck dissection in the N0 irradiated neck. Arch Otolaryngol Head Neck Surg 2002;128:292-295. 